\title{
Análise clínica e epidemiológica de fraturas radiculares na dentição permanente em uma subpopulação brasileira
}

\author{
Orlando Aguirre GUEDES ${ }^{1}$; Helder Fernandes de OLIVEIRA'; Giulliano \\ Caixeta SERPA2; Ana Célia de Oliveira AMORIM³; Carolina Cintra GOMES ${ }^{4}$; \\ Diogo Rodrigues CRUVINEL 5 ; Daniel de Almeida DECURCIO ${ }^{6}$
}

\begin{abstract}
1 - Professor Associado de Endodontia do Curso de Odontologia, Centro Universitário de Anápolis (UniEVANGÉLICA). Especialista, Mestre e Doutor em Endodontia; 2 - Professor Adjunto de Endodontia do Curso de Odontologia, Centro Universitário de Anápolis (UniEVANGÉLICA). Mestre em Clínica Odontológica pela Faculdade de Odontologia, Universidade Federal de Goiás (FO/ UFG) e Doutorando em Clínica Odontológica pela FO/UFG; 3 - Graduada em Odontologia pela Universidade Federal de Goiás (UFG). Especialista em Endodontia pela Associação Brasileira de Odontologia - Seção de Goiás (ABO-GO); 4 - Professora Associada de Radiologia do Curso de Odontologia, Centro Universitário de Anápolis (UniEVANGÉLICA). Especialista, Mestre e Doutora em Radiologia; 5 - Professor Associado de Prótese Dentária do Curso de Odontologia, Centro Universitário de Anápolis (UniEVANGÉLICA). Especialista, Mestre e Doutor em Prótese Dentária; 6 - Professor Associado de Endodontia da Faculdade de Odontologia, Universidade Federal de Goiás (FO/UFG). Especialista, Mestre e Doutor em Endodontia.
\end{abstract}

\section{Resumo}

Objetivo: Investigar os aspectos clínicos e epidemiológicos da fratura radicular na dentição permanente. Material e Método: A amostra do estudo foi composta por pacientes atendidos no serviço de urgência odontológica da Faculdade de Odontologia da Universidade Federal de Goiás, entre os anos de 1990 e 2003. As seguintes informações foram coletadas dos registros odontológicos de cada paciente: gênero, idade, fator etiológico, distribuição sazonal, estágio de desenvolvimento radicular, grupo dentário, número de dentes fraturados, tempo entre o trauma e o atendimento, direção, localização e número de fraturas, local do acidente, traumatismo associado e tratamento realizado. Resultados: Observou-se elevada ocorrência de fraturas radiculares em indivíduos do gênero masculino $(60,86 \%)$ e com idade entre $11-20$ anos (43,48\%). O principal fator etiológico foi a queda $(56,52 \%)$. Elevado número de traumatismos foi evidenciado no outono $(47,82 \%)$, sendo o incisivo central superior $(85,19 \%)$ o dente mais comumente afetado. Grande proporção dos dentes traumatizados apresentava rizogênese completa (66,67\%). A maioria dos dentes apresentou fratura horizontal $(81,48 \%)$ e deslocamento do fragmento $(85,19 \%)$. Vinte e quatro dentes apresentaram fratura simples $(88,89 \%)$. Quatorze pacientes sofreram a fratura radicular em ambiente externo $(60,87 \%)$. A maioria dos pacientes teve atendimento tardio $(43,48 \%)$, sendo que em quinze pacientes a contenção foi realizada $(65,22 \%)$. O tratamento mais comumente executado foi o acompanhamento clínico e radiográfico (33,33\%). Conclusões: Verificou-se elevado número de fraturas radiculares em indivíduos do gênero masculino, com idade inferior a 20 anos, decorrentes de quedas e envolvendo principalmente dentes superiores anteriores.

Copyright @ 2021 Revista Odontológica do Brasil Central Esta obra está licenciada com uma licença Atribuição-NãoComercialCompartilhalgual 4.0 Internaciona (CC BY-NC-SA 4.0)

Recebido: 04/04/2 Aceito: $05 / 04 / 21$ Publicado: 15/06/21
PALAVRAS-CHAVE: Fratura. Dentição permanente. Epidemiologia.

Traumatismos dentários.

\section{AUTOR PARA CORRESPONDÊNCIA}

Orlando Aguirre Guedes

Centro Universitário de Anápolis (UniEVANGÉLICA)

Av. Universitária, Km 3,5, Cidade Universitária, Anápolis-G0, CEP:75083-515.

E-mail: orlandoaguedes@gmail.com 


\section{Introdução}

Traumatismos dentários (TD) representam um dos mais sérios problemas de saúde pública ${ }^{1,2}$. Estudos populacionais têm evidenciado o aumento significativo na prevalência dessas lesões, bem como, seu impacto negativo na qualidade de vida de crianças e adolescentes ${ }^{1,3-5}$.

A fratura radicular $(\mathrm{FR})$ é um tipo raro de $\mathrm{TD}^{6,7}$, com prevalência reportada na dentição permanente de 0,5 - 7\% $\%^{2,3,8,9}$. Esta pode ser definida como uma fratura que envolve dentina, cemento e pol$\mathrm{pa}^{3}$. Indivíduos do sexo masculino, com idade variando entre 1120 anos são os mais frequentemente envolvidos ${ }^{5,6,9}$ e o incisivo central superior o dente mais comumente afetado ${ }^{8-10}$. Embora os fatores etiológicos dos TDs variem em função da idade ${ }^{2}$, a maioria das FRs ocorre em função de quedas, atos de violência e da colisão de corpos estranhos que atingem os dentes ${ }^{2,3,5}$.

A pós uma FR, são observados 4 padrões de cicatrização: 1) consolidação da fratura radicular através da interposição de tecido dentinóide e cementóide; 2) consolidação através da interposição de tecidos conjuntivo e ósseo; 3) consolidação através da interposição de tecido ósseo; e 4) não cicatrização devido a interposição de tecido conjuntivo ${ }^{11,12}$. Este último pode estar associado à necrose do tecido pulpar localizado no fragmento coronário, e ser uma indicação para a realização da terapia endodôntica ${ }^{8}$. Inúmeros fatores podem influenciar o tipo de cicatrização pós-fratura, como por exemplo, o intervalo de tempo decorrido entre o acidente e o primeiro atendimento, o estágio de formação radicular, o tipo de estabilização e o tipo e a localização da $\mathrm{FR}^{6,13,14}$.

O planejamento de políticas públicas com enfoque na prevenção dos incidentes que resultam em FRs, bem como em seu tratamento, deve ser baseado no conhecimento regional dos principais fatores de risco envolvidos ${ }^{5}$. A partir de evidências do reduzido número de estudos epidemiológicos na população brasileira $^{2,5}$ e, por considerar as especificidades e diferenças 
demográficas, culturais e socioeconômicas, se torna justificável analisar os aspectos clínicos e epidemiológicos das FRs na dentição permanente em pacientes tratados em um serviço de urgência odontológica.

\section{Material e método}

Este estudo transversal, descritivo e retrospectivo envolveu pacientes atendidos no serviço de urgência da Faculdade de Odontologia da Universidade Federal de Goiás, Goiânia (latitude $16^{\circ} 43^{\prime}$ S; longitude 4920’ W), Brasil e com histórico de FR em dentes permanentes. A busca foi realizada a partir de prontuários odontológicos obtidos no período compreendido entre 1990 e 2003.

Os dados relativos aos aspectos clínicos e epidemiológicos da FR (Figura 1) foram coletados dos registros odontológicos, fotográficos e radiográficos dos pacientes, com o auxílio de planilhas digitais, por dois estudantes de pós-graduação. Previamente à coleta dos dados, um estudo piloto foi realizado para testar a viabilidade e calibrar os examinadores com relação aos critérios utilizados. Foram excluídos do estudo casos envolvendo dentição decídua e com acompanhamento clínico e radiográfico inferior a um ano.

O protocolo do estudo foi aprovado pelo Comitê de Ética em Pesquisa da Universidade Federal de Goiás (Protocolo $\mathrm{n}^{\circ}$ 055/2005).

A análise estatística dos dados foi realizada com o programa SPSS for Windows 19.0 (SPSS Inc., Chicago, IL, EUA) e incluiu distribuição de frequência e teste de associação. A significância estatística para a associação entre as variáveis foi determinada pelos testes qui-quadrado e exato de Fischer. O nível de significância estabelecido foi de $\mathrm{p}<0,05$. 


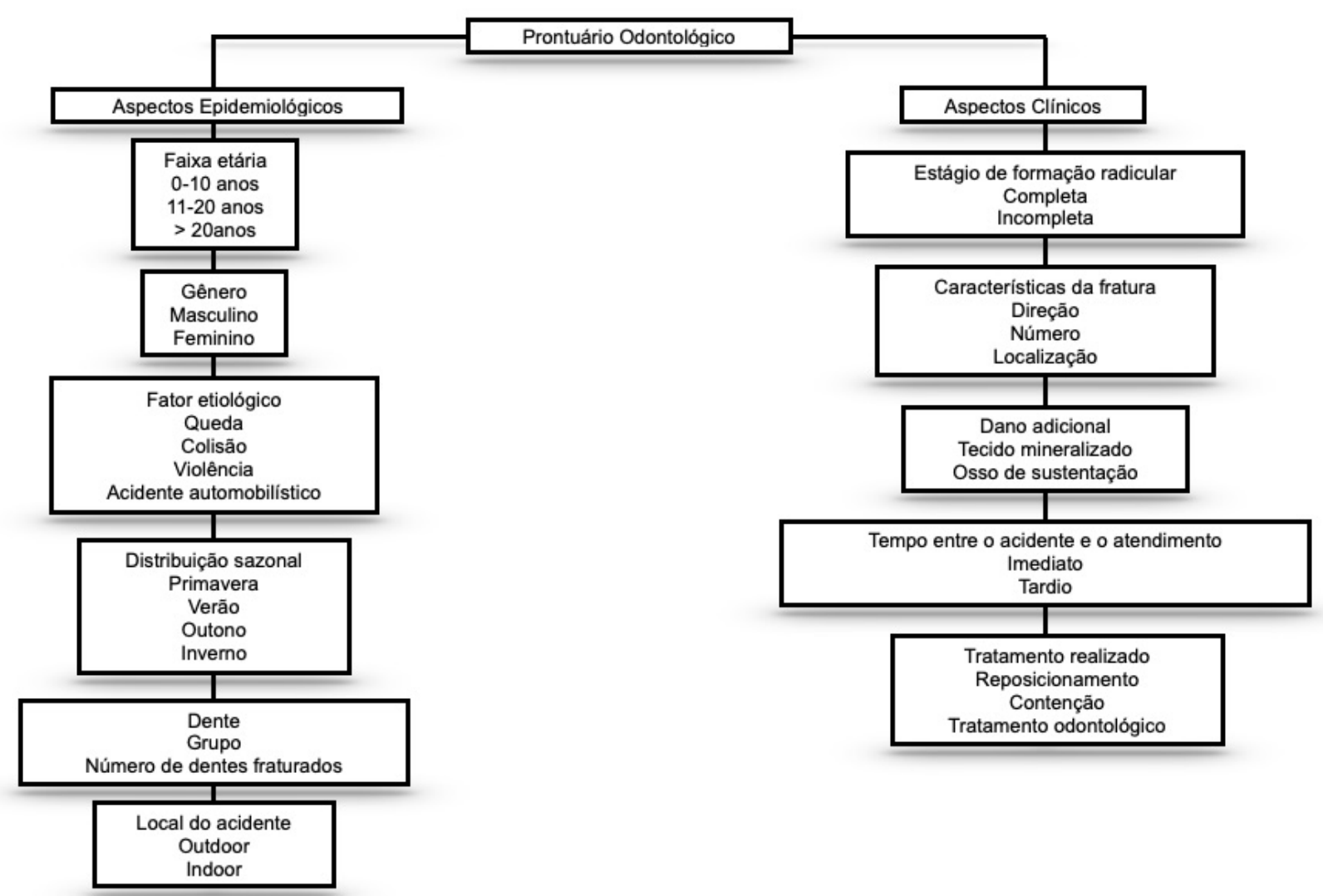

FIGURA 1 - Informações coletadas dos registros odontológicos, fotográficos e das radiografias dos pacientes.

\section{Resultados}

Faixa etária e gênero

A análise envolveu 23 pacientes com histórico de FR, com idade variando entre 8 e 34 anos (média de 17,4 anos). As maiores frequências foram registradas nos participantes de 11-20 $(\mathrm{n}=10$, $43,48 \%)$ e $0-10$ anos de idade ( $n=6,26,09 \%)$, seguido dos participantes maiores de 20 anos de idade ( $\mathrm{n}=6,26,09 \%)$. Do total de registros analisados, $14(60,86 \%)$ eram do gênero masculino e 9 $(39,13 \%)$ do gênero feminino, sendo observada uma proporção entre homens e mulheres de 1,55:1.

\section{Fator etiológico}

Treze pacientes $(56,52 \%)$ sofreram fratura radicular devido a quedas, cinco por colisão $(21,74 \%)$, dois por acidente automobilístico $(8,7 \%)$, dois por violência $(8,7 \%)$ e em um paciente não se obteve a causa $(4,35 \%)$. 


\section{Distribuição sazonal}

A distribuição sazonal revelou que a maioria dos casos ocorreram no outono (março a junho; $47,82 \%$ ), seguido pela primavera (junho a setembro; 30,43\%), verão (setembro a dezembro; 13,0\%) e inverno (dezembro a março; 8,69\%).

\section{Grupo dentário e número de dentes fraturados}

Os pacientes apresentaram um total de 27 dentes permanentes com FR, representando cerca de 1,5 dentes fraturados por acidente. Vinte e cinco (92,59\%) localizavam-se na maxila e $2(7,41 \%)$ na mandíbula. Os dentes mais comumente acometidos foram os incisivos centrais superiores (85,19\%), seguidos pelos incisivos laterais e caninos inferiores e caninos superiores $(7,40 \%$, cada um). Dezenove participantes $(82,61 \%)$ apresentaram o envolvimento de apenas um dente e $4(17,39 \%)$ de dois.

\section{Local da fratura}

Quatorze pacientes sofreram a FR em ambiente externo (60,87\%), seis em ambiente interno (26,09\%) e não foi possível obter a informação sobre o local do acidente em três pacientes $(13,04 \%)$.

\section{Estágio de formação radicular}

Dezoito dentes apresentavam rizogênese completa $(66,67 \%)$ e nove incompleta $(39,13 \%)$.

\section{Característica da fratura radicular}

Vinte e dois dentes apresentaram fratura radicular horizontal $(81,5 \%)$, seguidos de fratura oblíqua $4(14,8 \%)$ e não foi possível obter a informação de um dente (3,7\%).

Vinte e três dentes apresentaram deslocamento do fragmento $(85,19 \%)$, três não apresentaram $(11,11 \%)$ e não foi possível obter esta informação de um dente (3,7\%). 
Vinte e quatro dentes apresentaram fratura radicular simples $(88,89 \%)$, dois múltiplas $(7,41 \%)$ e não foi possível obter a informação de um dente $(3,7 \%)$.

Em doze dentes a fratura radicular localizou-se no terço apical $(44,44 \%)$, seguidos por 11 dentes no terço médio (40,73\%), dois no terço cervical $(7,41 \%)$, um nos terços cervical e apical $(3,7 \%)$ e um sem informação $(3,7 \%)$.

\section{Dano associado}

Vinte e um dentes não apresentaram traumatismos associados $(77,78 \%)$, dois sofreram avulsão do fragmento coronário $(7,41 \%)$, dois sofreram subluxação $(7,41 \%)$, um sofreu luxação extrusiva $(3,7 \%)$ e um não foi possível obter informação $(3,7 \%)$.

\section{Tempo entre o trauma e o atendimento}

Nesse caso, denominou-se atendimento imediato àquele que foi realizado até 30 minutos após o trauma. Dez pacientes tiveram atendimento tardio (43,48\%), e quatro o atendimento foi imediato $(17,39 \%)$. Em $39,13 \%$ da amostra não possível obter a informação com relação ao período de tempo entre o evento traumático e o atendimento odontológico.

\section{Tratamento realizado}

Em treze dentes não houve a redução da fratura (48,15\%), em doze ela ocorreu $(44,44 \%)$ e não foi possível obter a informação de dois dentes (7,41\%). Em quinze pacientes a contenção foi realizada $(65,22 \%)$, em seis a mesma não foi realizada $(26,09 \%)$ e dois não foi possível obter esta informação $(8,7 \%)$.

Com relação ao tipo de contenção realizada, os registros da grande maioria dos pacientes $15(65,22 \%)$ não apresentavam esse dado, em 5 a contenção semirrígida foi realizada (21,74\%) e em três foi aplicada a contenção rígida (13,04\%). 
A modalidade de tratamento mais frequente foi o acompanhamento clínico e radiográfico ( $n=9 ; 33,33 \%)$, seguido do tratamento endodôntico com trocas de medicação intracanal com hidróxido de cálcio + extração $(n=5 ; 15,52 \%)$, tratamento endodôntico com trocas de medicação intracanal com hidróxido de cálcio $(\mathrm{n}=4 ; 14,81 \%)$, acompanhamento clínico e radiográfico + posterior extração ( $n=2 ; 7,41 \%)$, acompanhamento clínico e radiográfico + remoção do fragmento apical + tratamento ortodôntico $(\mathrm{n}=1 ; 3,7 \%)$, remoção do fragmento apical + tratamento endodôntico com trocas de medicação intracanal com hidróxido de cálcio + obturação + restauração + extração + tratamento ortodôntico $(n=1 ; 3,7 \%)$, tratamento endodôntico com trocas de medicação intracanal do canal radicular com hidróxido de cálcio + remoção do fragmento apical + exodontia + tratamento endodôntico com obturação definitiva do canal radicular fora da boca + colagem dos fragmentos + reimplante + extração $(n=1 ; 3,7 \%)$, tratamento endodôntico com trocas de medicação intracanal do canal radicular com hidróxido de cálcio + remoção do fragmento apical ( $\mathrm{n}=1 ; 3,7 \%)$, tratamento endodôntico com trocas de medicação intracanal do canal radicular com hidróxido de cálcio + remoção do fragmento apical + obturação + restauração $(n=1$; $3,7 \%$ ), tratamento endodôntico com trocas de medicação intracanal do canal radicular com hidróxido de cálcio + obturação + restauração $(n=1 ; 3,7 \%)$, tratamento endodôntico com trocas de medicação intracanal do canal radicular com hidróxido de cálcio + pino intracanal + restauração $(n=1 ; 3,7 \%)$.

Não foram observadas diferenças estatisticamente significantes entre a ocorrência de FRs e os dados coletados dos registros dos pacientes $(p>0,05)$.

\section{Discussão}

O conhecimento dos fatores clínicos em associação às observações epidemiológicas é importante em função de algumas implicações, como por exemplo, o processo de cicatrização das FR 
e as possíveis complicações pós-traumáticas ${ }^{3,15}$. Não obstante, o entendimento dos mecanismos biológicos envolvidos torna-se fundamental durante a escolha da melhor conduta terapêutica a ser instituída ${ }^{5}$.

A análise do presente estudo foi retrospectiva, baseada na verificação de registros odontológicos, fotográficos e radiográficos de pacientes com FR e atendidos no serviço de urgência da Faculdade de Odontologia da Universidade Federal de Goiás, na cidade de Goiânia, no período compreendido entre 1990 a 2003. A população atendida pelo serviço pertencia a setores com baixo nível socioeconômico. Estudos transversais realizados em diferentes populações serviram de suporte ao presente trabalho ${ }^{5,8-10}$.

Do ponto de vista epidemiológico, os resultados do presente estudo estão em concordância com os dados apresentados previamente sobre a FR na dentição permanente ${ }^{6,9}$. Os dados coletados confirmam que indivíduos do gênero masculino sofrem mais FR do que os do gênero feminino (1,55:1). Em geral, meninos estão mais envolvidos em atividades de maior contato físico e ainda sem a utilização de proteção adequada ${ }^{1,4,6}$. No entanto, pesquisas têm observado índices cada vez maiores de casos de TD em indivíduos do gênero feminino, e têm justificado esses valores a uma maior participação das mulheres na sociedade ${ }^{16}$.

No presente estudo, a idade dos participantes com histórico de FR variou de 8 a 34 anos. Elevada prevalência foi observada no grupo de 11 a 20 anos (43,48\%), o que está em concordância com resultados de Andreasen et al. ${ }^{3}(2007)$. Molina et al. ${ }^{6}(2008)$ identificaram elevada prevalência de FR em crianças de 6-10 anos (95\%), enquanto Rajab ${ }^{17}(2003)$, no grupo etário de 10-15 anos. Vale a pena destacar a necessidade de cautela ao se comparar prevalências entre estudos que empregaram metodologias diferentes, visto a ausência de um padrão para definição da faixa etária estudada. 
As diferentes modalidades esportivas praticadas no mundo e as diferenças econômicas e culturais de cada população determinam a grande variação na frequência dos fatores etiológicos relacionados com a presença dos $\mathrm{TD}^{4}$. Os fatores causais identificados neste estudo estão em concordância com os observados em estudos desenvolvidos em outras populações ${ }^{5,6}$. A maioria das FR ocorreu em função de fatores não intencionais, como quedas $(56,52 \%)$ e colisões $(21,74 \%)$. Tem sido enfatizado que o lugar onde o estudo foi realizado e o grupo etário envolvido na amostra deve receber apropriada consideração durante a análise do fator etiológico dos $\mathrm{TD}^{2}$.

Apesar de algumas investigações indicarem as férias escolares e o verão como os períodos de maior incidência das lesões traumáticas ${ }^{18,19}$, os resultados do presente estudo não mostraram nenhuma das relações, sendo observada incidência elevada entre os meses de março e junho (outono; 47,82\%).

Quatorze pacientes sofreram FR em ambiente externo $(60,87 \%)$ e seis em ambiente interno (26,09\%). Majorana et al. ${ }^{9}(2002)$ observaram que pacientes em idade escolar apresentam o lar (62,5\%), a escola (25\%) o parque ou área de lazer $(12,5 \%)$ como os locais mais comuns para ocorrência de TD. Com relação aos adultos, os locais mais comuns são a via pública (34\%), local de trabalho e espaços para atividades desportivas (25\%). Panzarini et al. ${ }^{5}(2008)$ avaliaram os casos de TD envolvendo FR e/ou ligamento periodontal e notaram que os principais locais dos acidentes foram a via púbica (57\%) e o ambiente doméstico (14\%).

Os incisivos centrais superiores foram os dentes mais afetados, aspecto também observado previamente ${ }^{8,10}$. A posição vulnerável desse dente, que ainda pode se encontrar protruído e/ou com cobertura labial inadequada, podem explicar estes achados ${ }^{4}$.

No que se diz respeito à localização, o presente estudo constatou que a grande maioria das FRs estava localizada em terço apical $(44,44 \%)$, seguido pelos terços médio $(40,73 \%)$, e cervical $(7,41 \%)$, 
o que também foi observado por Andreasen e Hjørting-Hansen ${ }^{11}$ (1967), Zachrisson e Jacobsen ${ }^{20}$ (1975), Cvek et al. ${ }^{12}$ (2001) e Feely et al..$^{8}$ (2003). No entanto, Molina et al..$^{6}(2008)$ observaram taxas semelhantes de FR entre os terços médio e apical, enquanto Andreasen et al. ${ }^{21}$ (1989), Welbury et al. ${ }^{22}$ (2002) e Panzarini et al. ${ }^{5}$ (2008) reportaram maior frequência de FR em terço médio.

O tratamento de urgência das FRS, usualmente, é dirigido para o reposicionamento, estabilização do fragmento coronário na sua posição correta e monitoramento da vitalidade do dente ${ }^{13,14,23,24}$. Neste estudo 33,33\% dos pacientes atendidos receberam acompanhamento clínico e radiográfico. No que diz respeito ao tipo de estabilização utilizada, em $21,74 \%$ da amostra foi observado o emprego de contenções semirrígidas e em 13,04\% de contenções rígidas. Andreasen et al. ${ }^{13}$ (2004) observaram que nos casos de dentes com deslocamento dos fragmentos coronários, o uso de uma imobilização semirrígida resultou em maiores benefícios. Para os autores, com exceção das FR cervicais, que podem exigir contenções por períodos maiores, contenções por até 4 semanas são suficientes para assegurar a cura. O período de 4 semanas também tem sido recomendado pela Associação Internacional de Traumatologia Dentária (International Association of Dental Traumatology-IADT) em sua diretriz ${ }^{23}$. Para Molina et al. ${ }^{6}(2008)$, o reposicionamento ideal pode ser mais crítico do que a imobilização na obtenção de resultados satisfatórios. Cvek et al. ${ }^{12}$ (2001) não observaram diferença no padrão de cicatrização pós-fratura entre os dentes que receberam contenção e os que não receberam.

De acordo com a diretriz publicada ${ }^{23}$, basicamente, quatro modalidades terapêuticas têm sido descritas para o tratamento de dentes com FR: limpeza e obturação do fragmento coronário; limpeza e obturação de ambos os fragmentos com guta-percha; limpeza e obturação do fragmento coronário e a remoção cirúrgica do fragmento apical e tratamento endodôntico com trocas de medicação à base de hidróxido de cálcio seguido pela 
obturação $\mathrm{o}^{25-27}$. No presente estudo, várias modalidades de tratamento foram observadas tais como: tratamento endodôntico com trocas de medicação intracanal e posterior extração (15,52\%), acompanhamento clínico e radiográfico, remoção do fragmento apical, tratamento ortodôntico (3,7\%), tratamento endodôntico com trocas de medicação intracanal do canal radicular com hidróxido de cálcio, pino intracanal e restauração $(3,7 \%)$ e tratamento endodôntico com trocas de medicação intracanal (14,81\%). O elevado número de condutas terapêuticas encontrado ilustra o desafio vivido pelo clínico no restabelecimento da estética e da função em pacientes com crescimento facial incompleto e ressalta a necessidade de um conhecimento e de uma abordagem multidisciplinares.

A importância do presente estudo ocorre em função da ausência da análise clínica e epidemiológica da FR na dentição permanente na população de Goiânia-GO. Estudos prospectivos, baseados no acompanhamento destes pacientes, com vistas à avaliação dos protocolos terapêuticos e suas implicações necessitam ser desenvolvidos. No momento, o melhor prognóstico geral das FR sinaliza para a realização de campanhas preventivas como alternativa viável, funcional e operacional, indiferente ao sexo, faixa etária e nível socioeconômico, etc.

\section{Conclusão}

Baseado no estudo realizado pode-se concluir que:

Os aspectos clínicos e epidemiológicos da FR de dentes permanentes no serviço de urgência odontológica foram similares aos observados em estudos realizados em outras populações. Em que se verifica (i) elevado número de fraturas radiculares em indivíduos do sexo masculino, (ii) com idade inferior a 20 anos, (iii) decorrentes de quedas e (iv) envolvendo principalmente os dentes superiores anteriores. 


\section{Referências}

1 - Bastone EB, Freer TJ, McNamara JR. Epidemiology of dental trauma: a review of the literature. Aust Dent J. 2000; 45(1): 2-9.

2- Guedes OA, Alencar AH, Lopes LG, Pecora JD, Estrela C. A retrospective study of traumatic dental injuries in a Brazilian dental urgency service. Braz Dent J. 2010; 21(2): 153-7.

3 - Andreasen JO, Andreasen FM, Andersson L. Textbook and color atlas of traumatic injuries to the teeth. John Wiley \& Sons; 2018.

4 - Glendor U. Epidemiology of traumatic dental injuries: a 12 year review of the literature. Dent Traumatol. 2008; 24(6): 603-11.

5 - Panzarini SR, Pedrini D, Poi WR, Sonoda CK, Brandini DA, Castro JCM. Dental trauma involving root fracture and periodontal ligament injury: a 10-year retrospective study. Braz Oral Res. 2008; 22(3): 229-34.

6 - Molina JR, Vann Jr. WF, , McIntyre JD, Trope M, Lee JY. Root fractures in children and adolescents: diagnostic considerations. Dent Traumatol. 2008; 24(5): 503-9.

7 - Sheridan BA, Freccia WF, Silvestrin T, Bakland LK. Treatment options for permanent teeth with coronal one-third root fractures. J Am Dent Assoc. 2019; 150(3): 213-8.

8 - Feely L, Mackie IC, Macfarlane T. An investigation of root-fractured permanent incisor teeth in children. Dent Traumatol. 2003; 19(1): 52-4.

9- Majorana A, Pasini S, Bardellini E, Keller E. Clinical and epidemiological study of traumatic root fractures. Dent Traumatol. 2002; 18(2): 77-80.

10 - Yates JA. Root fractures in permanent teeth: a clinical review. Int Endod J. 1992; 25(3): 150-7.

11 - Andreasen JO, Hjorting-Hansen E. Intraalveolar root fractures: radiographic and histologic study of 50 cases. J Oral Surg. 1967; 25(5): 414-26.

12 - Cvek M, Andreasen JO, Borum MK. Healing of 208 intra-alveolar root fractures in patients aged 7-17 years. Dent Traumatol. 2001; 17(2): 53-62.

13 - Andreasen JO, Andreasen FM, Mejare I, Cvek M. Healing of 400 intraalveolar root fractures. 2. Effect of treatment factors such as treatment delay, repositioning, splinting type and period and antibiotics. Dent Traumatol. 2004; 20(4): 203-11.

14 - Andreasen JO, Andreasen FM, Mejare I, Cvek M. Healing of 400 intraalveolar root fractures. 1. Effect of pre-injury and injury factors such as sex, age, stage of root development, fracture type, location of fracture and severity of dislocation. Dent Traumatol. 2004; 20(4): 192-202.

15 - Cvek M, Mejare I, Andreasen JO. Healing and prognosis of teeth with intra-alveolar fractures involving the cervical part of the root. Dent Traumatol. 2002; 18(2): 57-65. 
16 - Gutmann JL, Gutmann MS. Cause, incidence, and prevention of trauma to teeth. Dent Clin North Am. 1995; 39(1): 1-13.

17 - Rajab LD. Traumatic dental injuries in children presenting for treatment at the Department of Pediatric Dentistry, Faculty of Dentistry, University of Jordan, 1997-2000. Dent Traumatol. 2003; 19(1): 6-11.

18 - Kargul B, Caglar E, Tanboga I. Dental trauma in Turkish children, Istanbul. Dent Traumatol. 2003; 19(2): 72-5.

19 - Tapias MA, Jimenez-Garcia R, Lamas F, Gil AA. Prevalence of traumatic crown fractures to permanent incisors in a childhood population: Mostoles, Spain. Dent Traumatol. 2003; 19(3): 119-22.

20 - Zachrisson BU, Jacobsen I. Long-term prognosis of 66 permanent anterior teeth with root fracture. Scand J Dent Res. 1975; 83(6): 345-54.

21 - Andreasen FM, Andreasen JO, Bayer T. Prognosis of root-fractured permanent incisors--prediction of healing modalities. Endod Dent Traumatol. 1989; 5(1): 11-22.

22 - Welbury R, Kinirons MJ, Day P, Humphreys K, Gregg TA. Outcomes for root-fractured permanent incisors: a retrospective study. Pediatr Dent. 2002; 24(2): 98-102.

23 - Diangelis AJ, Andreasen JO, Ebeleseder KA, Kenny DJ, Trope M, Sigurdsson A, et al. International Association of Dental Traumatology guidelines for the management of traumatic dental injuries: 1. Fractures and luxations of permanent teeth. Dent Traumatol. 2012; 28(1): 2-12.

24 - Andreasen JO, Ahrensburg SS, Tsilingaridis G. Root fractures: the influence of type of healing and location of fracture on tooth survival rates - an analysis of 492 cases. Dent Traumatol. 2012; 28(5): 404-9.

25 - Lindhl B. Transverse intra-alveolar root fractures. Roentgen diagnosis and prognosis Odontol Rev. 1958; 9:10-4.

26 - Michanowicz AE, Michanowicz JP, Abou-Rass M. Cementogenic rpair of root fractures. J Am Dent Assoc. 1971; 82(3): 569-79.

27 - Michanowicz AE. Root fractures. A report of radiographic healing after endodontic treatment. Oral Surg Oral Med Oral Pathol. 1963; 16: 1242-8. 


\title{
Clinical and epidemiological analysis of root fractures in permanent dentition in a brazilian subpopulation
}

\begin{abstract}
Objective: To investigate clinical and epidemiological aspects of root fracture in the permanent teeth. Material and Methods: The study sample consisted of patients seen at the Dental School of the Federal University of Goiás from 1990 to 2003. The following information was collected from each patient's files: gender, age, cause of injury, month when the root fracture occurred, root maturation level, permanent tooth type, number of fractured teeth, the time between the trauma and the care, direction, location and number of fractures, the environment in which the fracture has occurred, additional damage, and treatment. Results: The highest incidence was found in males $(60.86 \%)$ and aged $11-20$ years old $(43.48 \%)$. The main etiologic factors was fall (56.52\%). Most cases occurred in autumn (47.82\%) and the most affected tooth was the upper central incisor (85.19\%). A high proportion (66.67\%) of injured teeth had a completely formed root apex. Most teeth (81.48\%) had a horizontal fracture and displacement of the fragment (85.19\%). Twenty-three teeth showed fragment displacement (85.19\%). Twenty-four teeth had a simple fracture $(88.89 \%)$. Fourteen patients underwent a root fracture in an outdoor environment (60.87\%). Most patients had delayed service (43.48\%). Fifteen patients received tooth splinting (65.22\%). The most frequent treatment was clinical and radiographic follow-up (33.33\%). Conclusion: A high number of dental avulsions in males, aged under 20 years, from falls and mainly involving anterior maxillary teeth.
\end{abstract}

KEYWORDS: Fracture. Permanent teeth. Epidemiology. Tooth injuries.

\section{Como citar este artigo}

Guedes OA, Oliveira HF, Serpa GC, Amorim ACO, Gomes CC, Cruvinel DR. Decurcio DA. Análise clínica e epidemiológica de fraturas radiculares na dentição permanente em uma subpopulação brasileira. Rev Odontol Bras Central 2021; 30(89): 246-259. DOl: 10.36065/robrac.v30i89.1523 